\section{Resúmenes de trabajos libres del XII Encuentro Nacional de Medicina del Dormir}

\section{Abstracts of the XII National Meeting of Sleep Medicine}

Costos del insomnio crónico asociado a trastornos anímicos: un estudio observacional en México

\author{
GI Torres-Granados,* \\ JM Martínez-Núñez, ${ }^{*}$ \\ A Barrera-Medina, ${ }^{\ddagger} \mathrm{C}$ Cruz-Cruz, ${ }^{*}$ \\ U Jiménez-Correa, ${ }^{\ddagger} \mathrm{L}$ Carvalho-Silva, ${ }^{*}$ \\ F López-Naranjo, ${ }^{*}$ R Santana-Miranda ${ }^{\ddagger}$
}

*Departamento de Sistemas Biológicos, UAM Xochimilco; ${ }^{\ddagger}$ Clínica de Trastornos del Sueño, UNAM.

Introducción: Los costos relacionados con el insomnio asociado a trastornos del estado de ánimo siguen siendo poco estudiados pese a su alta prevalencia e incidencia mundial asociada a la pandemia COVID-19. Objetivos: Determinar los costos directos e indirectos del insomnio asociado a trastorno anímico. Material y métodos: Estudio observacional prospectivo, incluyó pacientes entre 18 y 65 años, diagnosticados con insomnio crónico asociado a trastornos del estado de ánimo (depresión y/o ansiedad) entre febrero de 2019 y febrero de 2020. Los datos sobre utilización de recursos sanitarios se recopilaron mediante la revisión de los registros médicos. Se diseñó un cuestionario para evaluar los costos no médicos directos e indirectos con datos sociodemográficos, transporte, bebidas energéticas, gastos en actividad física, modificaciones del hogar, compra de accesorios especiales, jornada laboral y salario. El ausentismo y el presentismo laboral se determinaron utilizando el Cuestionario de Salud y Desempeño de la OMS. Se realizó un análisis de sensibilidad determinista. Resultados: $\mathrm{EI}$ costo total estimado por paciente durante el primer año de tratamiento fue de 3,503.49 USD. La pérdida de productividad laboral fue el costo que más contribuyó a la carga económica (64.5\%), seguido de los costos médicos directos (27.6\%), mientras que los costos no médicos directos contribuyeron menos (7.9\%). Se calculó una carga económica anual de poco más de 290 millones de dólares para los pacientes tratados en el sistema de salud privado similar a la CTSUNAM. El análisis de sensibilidad mostró que la prevalencia de insomnio crónico asociado a trastorno del estado de ánimo, la pérdida de productividad laboral y el porcentaje de personas que buscan atención en un sistema privado fueron los parámetros con mayor impacto sobre la estimación de la carga económica total. Conclusiones: Los costos del insomnio asociado a trastorno anímico son altos.

Alteraciones de sueño y depresión en personas recuperadas de COVID-19

$$
\begin{aligned}
& \text { HA Suárez-Martínez, } \\
& \text { AD Santana-Vargas, *,* } \\
& \text { L Juárez-Santiago, *, G Millán-Rosas, }{ }^{*} \\
& \text { R Santana-Miranda* } \\
& \text { *Clínica de Trastornos del Sueño, UNAM; } \\
& { }^{\ddagger} \mathrm{HGM} \text {, «Eduardo Liceaga». }
\end{aligned}
$$

Introducción: La cuarentena domiciliaria ha aumentado la prevalencia de trastornos de sueño y alteraciones del estado de ánimo como la ansiedad y depresión. Estas alteraciones pueden ser mayores en las personas que desarrollaron y se recuperaron de COVID-19. Hipótesis: Las personas recuperadas de COVID-19 tendrán mayores alteraciones de sueño y del estado de ánimo que las que no han tenido COVID-19. Objetivo: Comparar los puntajes de cuestionarios de insomnio, calidad de sueño, ansiedad y depresión de personas que se han recuperado de COVID-19 con las que no lo han tenido. Material y métodos: Se aplicó el cuestionario Índice de Severidad de Insomnio (ISI), Índice de Calidad de Sueño de Pittsburgh (PSQI), Cuestionario de Salud del Paciente (PHQ-9) y la Escala de Ansiedad Generalizada (GAD-7) a personas que se recuperaron de COVID-19 en un lapso no mayor a dos semanas y a la población que no haya tenido COVID-19. Los cuestionarios se enviaron y contestaron por Internet, en el período de septiembre de 2020 a febrero de 2021. La muestra de estudio fueron pacientes que tuvieron cita virtual en la Clínica de Trastornos del Sueño de la UNAM. Se compararon las medianas de ambos grupos con la prueba $U$ de MannWhitney con significancia alfa menor a 0.05. Resultados: Participaron 72 personas recuperadas de COVID-19 (38 \pm 12.3 años, $61 \%$ mujeres) y 73 personas sin COVID-19 (36 \pm 12.7 años, $72 \%$ mujeres). La comparación entre los grupos fue significativa para el ISI $(11.5[5.25,16.7]$ versus $8[3,14]$, $p=0.027)$, el PSQI $(10[7.25,14]$ versus 9 $[4.5,14], \mathrm{p}=0.044)$ y PHQ-9 $(11[5.25,17]$ versus $7[3,12.5], p=0.016)$, sin diferencias en GAD-7 ( $p=0.071)$. Los puntajes fueron mayores para el grupo recuperado de COVID-19 que para los no-COVID-19. Conclusiones: Haber desarrollado y recuperado de COVID-19 incrementó la severidad del insomnio (ISI), la mala calidad de sueño (PSQI), así como la depresión (PHQ-9). Sin diferencias en la ansiedad.

\section{Perfil polisomnográfico en pacientes con depresión e insomnio con duración corta y normal de sueño}

JA González-Jaramillo, A Jiménez-Genchi,* C Olivera-López,* D Ortega-Robles, * A Díaz-Andaldúa*

*Clínica de Sueño, Instituto Nacional de Psiquiatría «Ramón de la Fuente Muñiz» (INPRFM).

Introducción: El insomnio es el trastorno del sueño más prevalente y está asociado con mayor riesgo de morbilidad psiquiátrica, principalmente con el trastorno de depresión mayor (TDM). Se han propuesto dos fenotipos basados en la duración objetiva del sueño: el de duración normal ( $\geq 6$ horas) con hiperalertamiento psicológico y el de duración corta ( $<6$ horas) con hiperalertamiento fisiológico, asociado a mayor vulnerabilidad biológica, y que parece representar la forma más grave de insomnio. Hipótesis: Los pacientes con TDM e insomnio con duración corta de sueño presentarán mayor alteración en la continuidad y arquitectura de sueño, en comparación con pacientes con insomnio con duración normal. Objetivo: Comparar continuidad y arquitectura de sueño en pa- 
cientes con TDM e insomnio con duración objetiva corta y normal de sueño. Material y métodos: Estudio transversal, comparativo. Se incluyeron pacientes ambulatorios de 18 a 60 años, con diagnóstico de TDM e insomnio en la Clínica de Sueño del INPRFM, sin tratamiento farmacológico y/o psicoterapéutico. Se realizó registro de dos noches de polisomnografía (PSG). Los parámetros de arquitectura y continuidad de sueño se tomaron de la primera noche, dividiendo a los participantes con base en el tiempo total de sueño (TTS): corta duración $<6$ horas y duración normal $\geq 6$ horas. El análisis estadístico se realizó con el programa SPSS 25; para la comparación entre los grupos se utilizó la prueba U de Mann-Whitney, el nivel de significancia se fijó con una $\mathrm{p}<0.05$. Resultados: Se incluyeron 27 pacientes, de los cuales 11 (40.74\%) conformaron el grupo con duración corta y 16 (59.25\%) el grupo con duración normal. Los pacientes con duración corta de sueño presentaron significativamente menor TTS $(4.95 \pm 0.80 \mathrm{~h}$ versus $6.75 \pm 0.44 h, p<0.01)$, eficiencia de sueño $(66.02 \pm 13.04 \%$ versus $84 \pm 5.05 \%$, $\mathrm{p}<0.01), \%$ MOR $(14.10 \pm 5.58$ versus $20.12 \pm 4.30, p=0.008$ ), y un aumento significativo en el tiempo despierto después del inicio de sueño (WASO) (140.98 \pm 70.14 versus $56.78 \pm 26.27, \mathrm{p} \leq 0.01)$. Conclusiones: Los pacientes con TDM e insomnio con duración corta de sueño muestran más cambios en los parámetros de continuidad de sueño y modificación en la arquitectura de sueño con disminución en el sueño MOR. Estos resultados sugieren que el fenotipo de insomnio con duración corta de sueño exhibe un perfil polisomnográfico distinto.

\section{Hallazgos polisomnográficos y calidad de vida en pacientes con fibrosis quística}

\section{JX Morales-García, *,* \\ JL Carrillo-Alduenda, ${ }^{*}$ \\ ER García-Colín, ${ }^{\ddagger}$ J Santa-María Aldaco, * A Alejandre-García, ${ }^{\ddagger}$ C Garrido-Galindo ${ }^{\ddagger}$ \\ *Unidad de Medicina del Sueño, INER; "Neumología Pediátrica, INER.}

Introducción: La fibrosis quística (FQ) es una enfermedad genética recesiva, caracterizada por infección bacteriana crónica y bronquiectasias, lo que resulta en hipoxemia, hipercapnia e incremento en el trabajo respiratorio; tiene características que afectan el sueño como: tos crónica, dolor musculoesquelético, pobre calidad de sueño y somnolencia diurna excesiva que se reportan de manera frecuente por cuidadores y afectan la calidad de vida. Objetivo: Evaluar la asociación entre los hallazgos polisomnográficos y la calidad de vida en pacientes con FQ. Material y métodos: Estudio transversal prospectivo. Se incluyeron pacientes con FQ atendidos en el INER. Se aplicaron cuestionarios Epworth pediátrico, CFQ-R, la escala de alteraciones del sueño en la infancia de Bruni y la escala SiNQ-5, así como polisomnografía (PSG) basal con un equipo ALICE G3, TCCO2 con SENTEC. Se exploraron correlaciones entre parámetros de calidad de vida y hallazgos en PSG mediante Spearman en el programa STATA versión 12. Resultados: Se incluyeron seis pacientes, con un mínimo de edad de seis años y máximo de 22, 50\% eran hombres, todos resultaron positivos al cuestionario de alteraciones del sueño en la infancia de Bruni, no se encontró relación entre debilidad diafragmática e hipoventilación ( $r s=0.33$ y $p=0.51$ ), tampoco se encontró asociación entre eventos respiratorios y la calidad de vida, pero sí se encontró asociación entre CFQR y N3 (rs $=0.94$ y $p<0.001)$. Conclusiones: Los pacientes con FQ tienen alteraciones del sueño medibles por el cuestionario de Bruni, el hallazgo polisomnográfico más relevante fue la hipoxemia durante el dormir y algunos presentan apnea obstructiva del sueño e hipoventilación nocturna; los dominios social e imagen corporal de calidad de vida por CFRQ tienen una asociación con sueño N3.

\section{FOSQ, ESE, PSQI, ISI, Beck y NOSE en 199 pacientes con SAOS del sureste mexicano

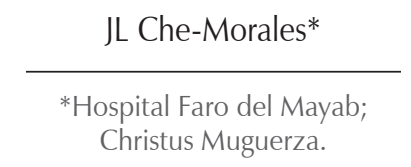

Introducción: El síndrome de apnea obstructiva del sueño (SAOS) es un padecimiento con impacto negativo en distintas esferas de la salud. Existe poca información en lo referente a las asociaciones y correlaciones entre somnolencia diurna excesiva (ESE), calidad de sueño (PSQI), insomnio (ISI), depresión (Beck), síntomas nasales (escala NOSE) y las consecuencias funcionales del dormir (FOSQ) en pacientes con SAOS en México. Objetivo: Conocer la asociación y correlación entre FOSQ, ESE, PSQI, ISI, Beck y NOSE en pacientes con SAOS. Material y métodos: Se analizó una muestra de 199 pacientes mayores de 18 años provenientes de seis estados del sureste de México, con diagnóstico de SAOS por monitor tipo III (Sleepware G3, 2016 Koninklijke, Philips N.V. ${ }^{\circledR}$ ) de 2018 a 2020 (número de registro: CEIS del CEM_2018_014). Se recabaron datos demográficos, poligráficos, cuestionarios FOSQ, ESE, PSQI, ISI, Beck y NOSE. Se calcularon correlaciones Pearson entre cuestionarios y se analizó mediante regresión logística uni- y multivariada los factores asociados a impacto funcional (FOSQ < 89.5) con el paquete estadístico SPSS versión 25.0. Resultados: El 52\% de los casos tuvieron SAOS grave. LoS puntajes en las diferentes escalas no mostraron correlación con el índice de eventos respiratorios. El FOSQ tuvo correlaciones negativas significativas ( $p<0.001)$ con ESE $(r=-0.57)$, PSQI $(r=-0.42)$, ISI $(r=-0.56)$, Beck $(r=-0.55)$ y NOSE $(r=-0.25)$. En el análisis univariado, las escalas confirieron riesgo de tener un puntaje global de FOSQ < 89.5; ESE > 10 puntos (OR: 4.4; IC95\% 2.4-8.2); PSQI > 5 puntos (OR: 4; IC95\% 1.9-8.5); ISI > 8 puntos (OR: 4.5; IC95\% 2.2-9.2) y Beck > 10 puntos (OR: 6.9; IC95\% 3.6-13.3). Sin embargo, la escala NOSE < 25 se manifestó como un factor protector (OR: 0.35; IC95\% 0.19-0.63). En el análisis multivariado los factores asociados en forma independiente con un puntaje de FOSQ < 89.5 fueron: edad > 65 años (OR: 3.1; IC95\% 1.2-7.9), ESE > 10 puntos (OR: 4.5; IC95\% 2-9.6) y Beck > 10 puntos (OR: 7.5; IC95\% 3.5-16.2). Conclusiones: Los puntajes de ESE, PSQI, ISI, Beck y NOSE mostraron una correlación negativa con FOSQ; la ESE, Beck y la edad > 65 años confirieron un riesgo incrementado de tener un impacto significativo en las consecuencias funcionales del dormir.

\section{Alteraciones de sueño y depresión en niños durante la cuarentena por COVID-19}

\footnotetext{
EE Godoy-de Dahbura, ${ }^{*}$ AD Santana-Vargas, *, HM Reyes-Obispo, G Millán-Rosas,* R Santana-Miranda*

*Clínica de Trastornos del Sueño, UNAM; ${ }^{\ddagger}$ Hospital General de México «Eduardo Liceaga».
} 
Introducción: Las condiciones de encierro dadas por la pandemia de COVID-19 han afectado a las poblaciones más vulnerables; los niños han sido propensos a desarrollar trastornos del sueño y del estado de ánimo, principalmente ansiedad y depresión. Un sesgo es preguntarles a los adultos que conviven con los niños en lugar de preguntarles a ellos mismos su percepción utilizando instrumentos adecuados. Hipótesis: Los niños que presenten depresión tendrán mayores alteraciones del sueño que los que no tengan depresión durante el confinamiento por la COVID-19. Objetivo: Detectar alteraciones del sueño y depresión mediante instrumentos diseñados para niños. Material y métodos: Se aplicó el cuestionario de alteraciones del sueño en niños escolares (EASE) y el cuestionario de depresión infantil (CDI) a niños de entre 6 y 12 años de edad, los cuales se contactaron vía Internet mediante la aceptación del consentimiento informado de sus padres y asentimiento por parte de los niños. Se compararon los grupos con y sin depresión de acuerdo al puntaje del $\mathrm{CDI}$, se realizaron pruebas de asociación y riesgo (OR) con el grupo no deprimido como referencia con la prueba $\chi^{2}$ con significancia alfa menor al 0.05. Resultados: Participaron 240 niños de $9.93 \pm 1.8$ años de edad $(n=111,46.3 \%$ niñas) que respondieron el $\mathrm{CDI}$, se encontraron 136 infantes con depresión (56.7\%) y 104 sin depresión (43.3\%). Los niños con depresión presentan dificultades para conciliar el sueño en $59.6 \%$ (OR: $3.4, \mathrm{p}=0.0001)$, pesadillas en $24 \%$ (OR: 8.17, $p=0.0001$ ), somnolencia diurna en 9.6\% (OR: 4.35, $p=0.012$ ), cansancio y dificultades para despertar en $47.1 \%$ (OR: 5.34, $p=0.0001)$. No se encontraron diferencias para el reporte de sonambulismo o despertares nocturnos. Conclusiones: Las condiciones de la pandemia han afectado el estado de ánimo de los niños, presentando depresión en casi 57\%. La depresión incrementa el riesgo de síntomas de sueño como dificultad para conciliar el sueño, pesadillas, somnolencia diurna y cansancio con dificultades para despertar.

Trastornos respiratorios del dormir en pacientes con discinesia ciliar primaria

LC Rivas-Hernández,

E García-Colín, ${ }^{*}$ AM Portillo-Vásquez,

F Partida-Cervantes, ${ }^{\ddagger} S$ Guerrero-Zúñiga ${ }^{\ddagger}$

*Neumología Pediátrica, INER; ${ }^{\ddagger}$ Unidad de Medicina del Sueño (UMS), INER.
Introducción: Los pacientes con discinesia ciliar primaria (DCP) tienen obstrucción de la vía aérea superior y bronquiectasias, alteración de la mecánica pulmonar e intercambio gaseoso; lo cual puede generar trastornos respiratorios del dormir (TRD). Objetivo: Describir síntomas, hallazgos polisomnográficos y trastornos del sueño en pacientes con DCP atendidos en el INER. Material y métodos: Estudio transversal prospectivo. Se incluyeron pacientes de 3-18 años con diagnóstico de DCP. Se llenó el cuestionario pediátrico de primera vez de la UMS y el Pediatric Sleep Questionnaire (PSQ, versión en español). Se realizó polisomnografía basal, equipo GRASS ${ }^{\circledR} 17$ canales, calificado manualmente según el manual de la AAMS versión 2.5. Se realizó estadística descriptiva según el tipo de variable y su distribución, en su mayoría mediana (P25-P75). Resultados: Se estudiaron 24 pacientes con cuestionario, de los cuales, 12 niños tenían edad de $9.7 \pm 3.5$ años. El tipo más frecuente de DCP fue el I. El 100\% reportó rinitis, $41.6 \%$ sobrepeso/obesidad, 33.5\% Mallampati III y 29.1\% hipertrofia amigdalina grado 3 y 4 . Según el PSQ 67\% de los niños presentó riesgo para síndrome de apnea obstructiva del sueño (SAOS) por puntaje $\geq 8$, los síntomas nocturnos más frecuentes fueron diaforesis y ronquido. Se realizaron 21 polisomnografías, con adecuada eficiencia de sueño, latencia a sueño y a sueño MOR. $\mathrm{SpO}_{2}$ promedio de 94\% (90-95\%) con nadir de $76 \%(68-82 \%)$. Tiempo de saturación < 90\% fue de 5\% (118\%). Presentaron índice de alertamientos de 6.3 e/h (5.8-9.2) y de movimiento periódico de extremidades (MPE) $2 \mathrm{e} / \mathrm{h}$ (0.9-4.4), índice de apneas e hipopneas de $3.2 \mathrm{e} / \mathrm{h}$ (1.4-5.2). Se encontraron 13 pacientes (61.9\%) con hipoxemia durante el sueño, 4 (19\%) presentaron MPE, 13 (61.9\%) con SAOS, 9 de intensidad leve y 4 moderada. Ninguno con hipoventilación. Conclusión: Los pacientes con DCP presentan alta prevalencia de síntomas de sueño, SAOS e hipoxemia durante el sueño respecto a la población pediátrica en general.

Aula virtual sobre apnea del sueño: una nueva estrategia de educar a los pacientes

\section{T Pérez-Gutiérrez, ${ }^{*}$ BN Orozco-González, D Hernández-Gordillo ${ }^{\ddagger}$}

*Neumología IMSS-CMNO; 'Departamento de Clínica de Sueño, IMSS-CMNO.
Introducción: El síndrome de apnea obstructiva del sueño (SAOS) tiene una prevalencia de $13 \%$ en población general. La presión positiva continua en vía aérea (CPAP) es el tratamiento de elección que ha demostrado reducción del riesgo cardiovascular cuando hay apego mayor al 70\%; para lograrlo, la educación al paciente puede mejorar la experiencia de uso. El Centro Médico Nacional de Occidente (CMNO) realizaba pláticas presenciales cada mes; sin embargo, la emergencia sanitaria por COVID-19 obligó a implementar un aula virtual. Objetivo: Describir la participación de los pacientes con SAOS en el aula virtual durante la pandemia de COVID-19. Material y métodos: Estudio transversal analítico, incluyó a pacientes con SAOS e indicación de CPAP, mayores de 18 años con expediente electrónico. Fueron contactados por vía telefónica para determinar interés en acudir a una plática virtual, los interesados autorizaron recibir la ruta de acceso, fecha y hora de la clase virtual. Las diapositivas fueron elaboradas por médicos especialistas con experiencia en clases presenciales, cada sesión duró 30 minutos a las 18:00. Al finalizar se les envió una encuesta que incluía disposición para continuar recibiendo clases virtuales. Fue aprobado por el comité de ética local (R-2020-1301-039). Se realizó estadística descriptiva según el tipo de variable, y se compararon mediante $\chi^{2}$ y t de Student, considerándose un valor de $p<0.05$ significativo. Se calculó razón de momios (OR) con intervalo de confianza del 95\%, en el programa Stata 9.2. Resultados: Se identificaron 159 pacientes que acudieron a consulta de enero de 2019 a marzo de 2020, 111 (69.8\%) contestaron y 81 (72.9\%) mostraron interés en acudir. Únicamente $46(56.7 \%)$ acudieron a la plática virtual. Los pacientes que acudieron fueron 29 hombres (63\%), 31 con edad mayor a 55 años (67.4\%), 71.8\% tenían más de dos comorbilidades, Epworth 12.6 ( \pm 6.12) e IAH de 45.8 ( \pm 18.9). Los mayores de 55 años se conectaron más con un OR de 3.4 (1.2-9.7), y aquéllos invitados por consulta y llamada telefónica asistieron más que los invitados por vía telefónica OR 6.5 (1.1565.9). El $76 \%$ contestó la encuesta y todos respondieron que les gustaría continuar con las clases virtuales. Conclusiones: El $41 \%$ de los pacientes contactados por vía telefónica asisten al aula virtual, existe mayor participación en los mayores de 55 años y aquéllos invitados por consulta 
y llamada telefónica. La mayoría de los asistentes muestran interés por continuar esta modalidad, por lo que consideramos puede ser una estrategia aceptable para implementar de manera permanente.

Experiencia en el uso del sistema de Telesalud en la Clínica de Trastornos del Sueño de la UNAM, a partir de la pandemia por COVID-19

\section{YK Camez-Escalante, * G Millán-Rosas,* AD Santana-Vargas,* A Acosta-Bolívar,* R Santana-Miranda*}

*Clínica de Trastornos del Sueño, UNAM.

Introducción: El confinamiento por la pandemia por la COVID-19 obstaculizó los servicios de asistencia sanitaria presencial en todo el mundo. La Clínica de Trastornos del Sueño (CTS) de la UNAM, implementó el Sistema de Telesalud, ante la necesidad de continuar con las actividades asistenciales, exacerbación de trastornos del sueño por la contingencia y previamente en la comunidad universitaria en el extranjero. La telesalud se refiere a la incorporación de las tecnologías de la información y la comunicación en los sistemas de salud, con el propósito de intercambiar información, permitiendo solucionar barreras geográficas y mejorar la accesibilidad. Objetivo: Medir la satisfacción de los pacientes atendidos en la consulta a través del Sistema de Telesalud y de los especialistas que los atienden en la CTS de la UNAM, en el período entre julio y octubre de 2020. Material y métodos: Se invitó a los pacientes y especialistas de la CTS de la UNAM, usuarios del Sistema de Telesalud, a participar en el estudio por correo electrónico donde se explicó el objetivo del estudio y se adjuntaron el consentimiento informado y la encuesta de satisfacción con opciones de respuesta «excelente y muy de acuerdo» codificada como 5, «buena y de acuerdo» como 4, «regular y neutro» como 3, «mala y en desacuerdo» 2, «muy mala y muy en desacuerdo» 1. El análisis estadístico se realizó de forma independiente para los pacientes y especialistas. Se realizó el conteo y se promediaron los valores codificados de las respuestas. Resultados: Los pacientes calificaron su satisfacción con la teleconsulta con un promedio de 4.21 dentro del rango buena-satisfecho. Los especialistas calificaron en promedio con
3.96 correspondiente a satisfacción regular. En cuanto a los aspectos técnicos del sistema, la satisfacción de los pacientes fue de 4.29 (buena-satisfecho) y de 3.82 (regular) para los especialistas. Conclusiones: Los pacientes tienen buena satisfacción con la teleconsulta y los aspectos técnicos del Sistema de Telesalud, mientras que los especialistas se encontraron con satisfacción regular en ambos aspectos. Tanto pacientes como especialistas consideran muy factible continuar con el Sistema de Telesalud.

Asociación de polifarmacia, insomnio y funcionalidad en el adulto mayor

YK Camez-Escalante, * G Millán-Rosas, AD Santana-Vargas,* R Santana-Miranda*

*Clínica de Trastornos del Sueño, UNAM.

Introducción: El envejecimiento de la población representa un desafío para el sector salud. Es indispensable contar con una valoración geriátrica integral para identificar síndromes geriátricos; los cambios ontogenéticos del ciclo sueñovigilia, mayor prevalencia de múltiples comorbilidades, incluidos los trastornos del sueño, y la presencia de polifarmacia hace a este grupo más vulnerable a padecer insomnio crónico comórbido. Hipótesis: Existe una asociación entre la cantidad de fármacos, la severidad de insomnio y el deterioro funcional del paciente geriátrico. Objetivo: Determinar la asociación entre polifarmacia e insomnio y su impacto en la funcionalidad en adultos mayores. Material y métodos: Estudio observacional retrospectivo, se analizaron los expedientes de adultos mayores con insomnio y polifarmacia. Se consideró un modelo factorial completo con interacciones con tres factores y dos niveles por cada uno. Los factores fueron polifarmacia, deterioro funcional mayor e insomnio. Para la asociación se consideró un modelo de $2 \times 2 \times 2$. Se empleó la prueba de $\chi^{2}$ para el modelo completo y para los modelos reducidos de $2 \times 2$, todos con una significancia $\leq 0.5$. Las variables categóricas se analizaron mediante proporciones y pruebas no paramétricas para grupos independientes mediante la prueba $U$ de Mann-Whitney y para las cuantitativas medias y desviaciones estándar y la prueba t de Student para grupos independientes considerando el mismo nivel de significancia. Resultados: Se revisaron 366 expedientes de adultos mayores, 199 (54.4\%) con polifarmacia, los fármacos más frecuentes son ARA 2 (35.2\%), hipoglucemiantes orales (26.8\%) y herbolaria (27.9\%). Se encontró insomnio en 98 pacientes $(26.8 \%), 12 \%$ se encontraban en tratamiento con antidepresivos, $5.2 \%$ con benzodiacepinas y $4.4 \%$ con antipsicóticos. Conclusiones: La asociación de polifarmacia, insomnio y funcionalidad en el adulto mayor en nuestro estudio no fue concluyente, es necesario incrementar el tamaño de la muestra y ampliar la información sobre entidades nosológicas.

Patrones y factores asociados del uso irracional de medicamentos en pacientes con insomnio

\section{Carvalho-Silva,, JM Martínez-Núñez, U Jiménez-Correa, ${ }^{\S}$ C Cruz-Cruz, R Santana-Miranda, ${ }^{\S}$ K Sánchez-Herrera, GI Torres-Granados ${ }^{\ddagger}$}

\footnotetext{
*Maestría en Ciencias Farmacéuticas, UAM Xochimilco; ${ }^{\ddagger}$ Departamento de Sistemas Biológicos, UAM Xochimilco; ${ }^{\S}$ Clínica de Trastornos del Sueño, UNAM.
}

Introducción: El insomnio es el trastorno del sueño con mayor prevalencia en todo el mundo. En México, el insomnio fue la segunda patología del sueño más frecuente (36.9\%) en 2016. Éste es tratado farmacológicamente con hipnóticos no benzodiacepínicos (no-BZD), benzodiacepínicos (BZD) y otros medicamentos con efecto sedante. El uso irracional (UI) de estos medicamentos conlleva a un problema serio de salud pública, por lo tanto, es importante considerar los patrones y factores asociados a su uso irracional. Objetivo: Describir los patrones y los factores asociados con el UI de hipnóticos, antidepresivos y antipsicóticos en pacientes con insomnio atendidos en la CTS UNAM. Material y métodos: Se realizó un estudio transversal analítico, con información recolectada a través de expedientes clínicos y entrevistas directas a pacientes de primer ingreso de la CTS UNAM. La información fue analizada a través de estadística descriptiva y comparaciones con prueba de $\chi^{2}$, considerando un nivel de significancia de $\alpha<0.05$. Resultados: Se incluyeron 89 pacientes, 64\% fueron mujeres, 52\% tenían entre 60 y 79 años, $65 \%$ utilizaban hipnóticos (57\% BZD y $8 \%$ 
no-BZD). El clonazepam representó $70 \%$ de los benzodiacepínicos. Más de 93\% de los pacientes presentaron UI con al menos uno de los medicamentos que consumían. Los tipos de UI más frecuentes fueron de duración en $47 \%$ (más tiempo de lo que recomienda la literatura) y por indicación en $43 \%$ (terapéutica errónea de acuerdo a su diagnóstico). Los pacientes mayores de 60 años presentan más UI, las clases farmacéuticas con alto porcentaje de UI fueron BZD > 90.3\%; no-BZD > 62.5\%; APs $>83.3 \%$. El 94\% de los pacientes adquirieron su tratamiento con anterioridad a través de la prescripción de uno o más médicos. Las comorbilidades más frecuentes en los pacientes atendidos por insomnio fueron la hipertensión 26\%, diabetes $12 \%$ y depresión 12\%. Conclusión: Se observó un patrón de uso irracional de benzodiacepinas y otras clases farmacéuticas, principalmente de duración e indicación, es más frecuente en mayores de 60 años, la fuente de adquisición más prevalente fue «recetado a través de un médico». Es necesario elaborar una intervención educativa para pacientes de primer ingreso en la CTS, que hacen UI de medicamentos para el insomnio y que posean comorbilidades asociadas.

Impacto de la restricción de sueño sobre la cognición social en residentes de medicina

\section{B Jiménez-Ceballos, * J Velázquez-Moctezuma;* DY Arana-Lechuga,* GY Terán-Pérez*}

*UAM Iztapalapa.

Introducción: La restricción de sueño se define como la reducción del tiempo total de sueño que requiere un individuo; y tiene implicaciones cognitivas, conductuales, funcionales y endocrinas, entre otras. Pese a estas consecuencias negativas, las sociedades modernas continúan disminuyendo el tiempo que duermen y con ello incrementan sus efectos negativos. Uno de los factores sociales que restringen el sueño son los laborales con jornadas prolongadas o turnos rotatorios que impiden que el sujeto duerma las horas suficientes en condiciones adecuadas. Ante esta problemática social surge la necesidad de conocer las consecuencias de la restricción de sueño sobre la cognición social en trabajadores con jornadas prolongadas como los resi- dentes de medicina. Objetivo: Determinar el impacto de la restricción de sueño sobre funciones cognitivas en residentes y evaluar el impacto de proporcionar un curso taller de estrategias de mitigación para la restricción de sueño y mejorar el funcionamiento diurno. Material y métodos: Estudio longitudinal prospectivo, se evaluaron 124 residentes de medicina (74 mujeres) en cuatro momentos (preguardia, posguardia, preguardia después de la intervención y posguardia después de la intervención), por medio de pruebas cognitivas (MSCEIT, PVT, Depresión y Ansiedad de Beck) y cuestionarios de sueño (Atenas de insomnio, Epworth, Karolinska, STOP BANG, Pittsburgh, hábitos de sueño y diario de sueño). Para el análisis de los resultados se utilizó la prueba ANOVA para medidas repetidas de dos vías. Resultados: Se presentó mayor índice de deterioro en cognición social; estas diferencias se asociaron a restricción de sueño. Cuando los participantes se encontraron en restricción aguda (posguardia) se observaron puntuaciones significativamente más bajas. Además, se observó que los residentes llevaban a cabo hábitos inadecuados relacionados a su dormir. Aunque no lograron obtener puntajes dentro de lo esperado, se observaron diferencias estadísticamente significativas después de la intervención. Conclusión: La restricción aguda de sueño genera un impacto significativo en la cognición social que mejoran después de implementar estrategias de mitigación sin alcanzar valores normativos en residentes de medicina.

\section{Duración corta de sueño y alteraciones cognitivas en pacientes con depresión mayor e insomnio}

\section{Olivera-López,* A Jiménez-Genchi,* D Ortega-Robles*}

\section{*Clínica del Sueño, Instituto Nacional de Psiquiatría «Ramón de la Fuente Muñiz» (INPRFM).}

Introducción: El trastorno depresivo mayor (TDM) y el insomnio mantienen una relación bidireccional que ha sido poco estudiada. A pesar del avance en la comprensión de la neurobiología que comparten, se sabe poco de las consecuencias que genera presentar esta comorbilidad. Una de las quejas principales son las alteraciones en las funciones cognoscitivas. Los resultados han sido inconsistentes en pacientes con un solo trastorno y con pruebas neuropsicológicas poco sensibles. De manera reciente, se ha propuesto que la duración corta de sueño $(<6$ h) puede estar asociada al hiperalertamiento fisiológico y representar un fenotipo más severo del insomnio. Por lo que la duración objetiva del sueño puede ser el factor que vincule el TDM con el insomnio y las alteraciones cognoscitivas. Hipótesis: Los pacientes con TDM e insomnio que presenten una duración corta del sueño $(<6$ h) tendrán una disminución en el funcionamiento cognoscitivo comparados con la duración normal del sueño ( $\geq 6$ h). Objetivo: Comparar las funciones cognoscitivas entre pacientes con duración objetiva corta del sueño y duración normal que presentan TDM e insomnio. Material y métodos: Estudio transversal analítico, se incluyeron pacientes de nuevo ingreso a la clínica del sueño del INPRFM con diagnóstico de TDM e insomnio, sin abuso de sustancias ni tratamiento farmacológico, sin presencia de ronquido o sospecha de alguna otra alteración del sueño. Fueron evaluados con una batería de pruebas neuropsicológicas estandarizada y sensible, pruebas clinimétricas para determinar la severidad del insomnio, del TDM y descartar alguna otra alteración del sueño, los cuales fueron clasificados de acuerdo a su tiempo total de sueño en durmientes cortos $(<6 \mathrm{~h})$ y durmientes normales ( $\geq 6$ h) por polisomnografía. Resultados: Se incluyeron 23 pacientes, 12 mujeres con edad de $36.9 \pm 13.2$ años. A excepción de los años de estudio acumulados, no se encontraron diferencias significativas entre los grupos de duración corta y normal de sueño en las variables sociodemográficas. Las diferencias en las funciones cognoscitivas entre durmientes cortos y normales sugieren una disminución en la memoria episódica, memoria de trabajo, atención selectiva y planeación en pacientes con duración corta de sueño. Conclusión: Las diferencias entre los grupos sugieren que la duración objetiva de sueño tiene un impacto en el funcionamiento cognoscitivo y puede ser el vínculo entre el TDM con insomnio y las alteraciones cognoscitivas. 
Calidad de sueño en pacientes posCOVID del Nuevo Hospital General ISSSTE «Dra. Matilde Petra Montoya Lafragua»

\author{
CM Colin-Cano, *, \\ IA Fernández-Navarrete,* \\ B Pérez-Garduño, ${ }^{*}$ LI Ledo-Gutiérrez, \\ M Contreras-Granillo,* \\ FR Peña-Velázquez, ${ }^{*}$ M Canales-Jaime,* \\ LE Mendoza-Abarca,* \\ D Rodríguez-Pérez,* \\ JM Hernández-Salas*
}

\footnotetext{
*Nuevo Hospital General ISSSTE «Dra. Matilde Montoya Lafragua»; ${ }^{\ddagger}$ Centro de Atención Multidisciplinario y Análisis de Sueño
} (CAMAS).

Introducción: Los trastornos del dormir están presentes en gran parte de la población, tales como el insomnio, apnea del sueño, entre otros, los cuales se han visto modificados a causa del COVID-19, reportándose varias secuelas en su calidad de sueño después de la enfermedad, insomnio recurrente, desfase del sueño o fatiga extrema. Hipótesis: Existe una mala calidad de sueño en pacientes pos-COVID. Objetivo: Describir la calidad de sueño en pacientes pos-COVID. Material y métodos: Estudio trasversal, descriptivo. Se realizó una encuesta a 73 voluntarios que tuvieran prueba positiva de COVID-19 en el Nuevo Hospital General ISSSTE «Dra. Matilde Montoya Lafragua» para conocer la calidad del sueño y los tipos de afectaciones de la misma mediante el índice de calidad de sueño en computadora, tableta o celular, en la plataforma de cuestionarios Google, el análisis estadístico se realizó en el programa SPSS 21. Resultados: Cincuenta y cuatro punto siete por ciento de los infectados requirieron hospitalización, edad media de 49 años. El 50.7\% calificó su calidad de sueño con 7 a 8 de 10 puntos y $20 \%$ de 5-6; $89.3 \%$ reportó sensación de cansancio diurno, $77.3 \%$ sintió la necesidad de dormir durante el día, $68 \%$ reportó ronquido, $57.3 \%$ malestar general por haber dormido mal, $38.7 \%$ de la población se desvela de uno a dos días por semana y $36 \%$ despierta por lo menos una vez en la noche. Conclusiones: El síntoma más frecuente fue la sensación de cansancio diurno en $89.3 \%$ de la población a pesar de darle buena calificación a su dormir y no presentar otros síntomas que afecten el sueño.

Efecto del tratamiento con presión positiva en pacientes con síndrome de apnea/hipopnea obstructiva del sueño comórbido con insomnio

\section{AD Santana-Vargas, ${ }^{*}{ }^{\ddagger}$ CA Toledo-Triana, ${ }^{*}$ G Millán-Rosas,* R Santana-Miranda* \\ *Clínica de Trastornos del Sueño, UNAM; "HGM, «Eduardo Liceaga».}

Introducción: El insomnio y el síndrome de apnea hipopnea obstructiva del sueño (SAHOS) son las dos patologías más frecuentes de los trastornos del sueño, donde de 29 a $67 \%$ de los pacientes con insomnio tienen un índice de apnea hipopnea $(\mathrm{IAH})>5$. Además del insomnio se pueden presentar otras comorbilidades como depresión y ansiedad que a su vez pueden influir en la adherencia al tratamiento con presión positiva (CPAP) para el SAHOS, adicionalmente el efecto del CPAP sobre estas comorbilidades ha sido poco estudiado. Hipótesis: La depresión y el insomnio en pacientes con SAHOS pueden interactuar con la adherencia al CPAP y mejorar con el tratamiento en un período de seis meses. Objetivo: Evaluar la interacción entre el insomnio, la depresión, la calidad del sueño y la somnolencia en pacientes con SAHOS, insomnio y depresión con tratamiento con CPAP por seis meses. Material y métodos: Estudio longitudinal prospectivo, se incluyeron pacientes con SAHOS, comórbidos con insomnio y depresión en la Clínica de Trastornos del Sueño de la UNAM, se les aplicó al inicio del tratamiento con CPAP, al mes y seis meses las escalas: 1. Atenas de Insomnio, 2. Hamilton de depresión, 3. Somnolencia de Epworth y 4. Índice de calidad de sueño de Pittsburgh. Se realizó un análisis de varianza de medidas repetidas con el tiempo como factor intragrupos y apego al tratamiento como factor entre grupos, con un nivel de significancia alfa $<0.05$. Resultados: Se incluyeron 22 pacientes con media de edad $46.2 \pm 8.9$ años, $77.3 \%$ fueron hombres. El insomnio presentó interacción con la adherencia al CPAP ( $p=0.047)$. Se observó efecto estadísticamente significativo del tiempo de tratamiento con CPAP para el insomnio (Atenas $13.1 \pm 3.2$ versus $3.95 \pm 3.2, p<0.001$ ), depresión (Hamilton $15.6 \pm 6.4$ versus $9.72 \pm 3.8, p=0.002$ ), somnolencia (Epworth $14.5 \pm 4.8$ versus $5.0 \pm 4.7, \mathrm{p}<0.001)$ y calidad de sueño (Pittsburg $13.0 \pm 3.4$ versus $6.77 \pm 4.4$, $p<0.001)$. Conclusiones: El insomnio en pacientes comórbidos con SAHOS no es independiente al tratamiento con CPAP en función de la adherencia al tratamiento. El insomnio, la depresión, la calidad de sueño y la somnolencia mejoran con el tratamiento con el tiempo, pero de forma independiente a la adherencia. 